\title{
A direct variational approach to a problem arising in image reconstruction
}

\author{
LUIGI AmBrosio ${ }^{\dagger}$ \\ Scuola Normale Superiore, Piazza dei Cavalieri 7, 56126 Pisa, Italy \\ AND \\ SIMON MASNOU \\ Laboratoire Jacques-Louis Lions, B.C. 187, Université Pierre-et-Marie-Curie, \\ 75252 Paris Cedex 05, France
}

[Received 14 May 2002 and in revised form 31 August 2002]

We consider a variational approach to the problem of recovering missing parts in a panchromatic digital image. Representing the image by a scalar function $u$, we propose a model based on the relaxation of the energy

$$
\int|\nabla u|\left(\alpha+\beta\left|\operatorname{div} \frac{\nabla u}{|\nabla u|}\right|^{p}\right), \quad \alpha, \beta>0, p \geqslant 1,
$$

which takes into account the perimeter of the level sets of $u$ as well as the $\mathrm{L}^{p}$ norm of the mean curvature along their boundaries. We investigate the properties of this variational model and the existence of minimizing functions in $\mathrm{BV}$. We also address related issues for integral varifolds with generalized mean curvature in $\mathrm{L}^{p}$.

Keywords: Image processing; image reconstruction; BV; mean curvature; varifolds; relaxation.

\section{Introduction}

Many problems in digital image processing require the ability to recover missing parts of an image or to remove spurious or undesired objects. One can mention for instance the removal of scratches in old photographs and films, the recovery of pixel blocks corrupted during a binary transmission (or analogously the removal of impulse noise) or the removal of undesired publicity, text or subtitles from a photograph. One can also think of special effects for movie postproduction, e.g. the removal of a microphone appearing in a scene.

A digital image is usually modeled as a function $u$ from a bounded domain of $\mathbb{R}^{N}$ ( $N=2$ for usual snapshots, $N=3$ for medical images or movies, $N=4$ for moving medical images) onto $\mathbb{R}^{M}$ ( $M=1$ for a grey level image, $M=3$ for colour images). Since it is now well admitted that the essential features of any natural image are contained in its grey level representation, we shall concentrate on the panchromatic case $M=1$. To extend to the colour case an operator designed for grey level images, it is generally enough to process separately each channel in the colour representation, e.g. the red-green-blue representation or, more appropriately, any representation with

\footnotetext{
†Email: luigi@ambrosio.sns.it
}

Email: masnou@ann.jussieu.fr 
two channels for the chromaticity and one channel for the luminosity (see [9] and the references therein).

After the work of L. Rudin and S. Osher [34], the usual representation of a panchromatic image is a sum of two components $u_{1} \in \mathrm{BV}\left(\mathbb{R}^{N}\right)$ and $u_{2} \in \mathrm{L}^{2}\left(\mathbb{R}^{N}\right)$. The component $u_{1}$ is supposed to describe the geometry of the image, i.e. its objects and their boundaries, while $u_{2}$ contains all information about texture and additive noise. The assumption that the geometry of the image can be described by a function of bounded variation sounds quite natural, for it means that there can be discontinuities in the image but supported on rectifiable curves. The necessity of another component that does not necessarily belong to BV can be corroborated by an experimental procedure that seems to indicate that, given a digital image, the underlying "real" image may be often too oscillating to belong to BV (see [2] for the details and [11] for related theoretic issues). The reader may refer to [4, 20] for a detailed survey of the space BV.

Among the large literature that has been published in recent years on the recovery of missing parts in a digital image, one can basically distinguish between two approaches and each of them corresponds in some way to the processing of one component in the decomposition above:

- The stochastic approach, which is based on the modeling of an image as a realization of a random process. Usually, it is assumed that the image intensity derives from a Markov random field and, therefore, has the properties of locality and stationarity, i.e. each pixel is only related to a small set of neighboring pixels and different regions of the image are perceived similar. This modeling is particularly adapted for texture images (thus to the processing of the component $u_{2}$ in the previous decomposition) and has motivated numerous works on texture analysis and synthesis [5, 14, 15, 25, 32, 33, 42, 44].

- The deterministic approach, whose main purpose is to recover the geometry of the image. The model we shall discuss in this paper belongs to this category.

A pioneering work on the recovery of plane image geometry is due to D. Mumford, M. Nitzberg and T. Shiota [31]. They did not directly address the problem of recovering missing parts in an image but rather tried to identify occluding and occluded objects in order to compute the image depth map. Their algorithm starts with the detection of the boundaries of image objects. The next step is the identification of occluded and occluding objects. To this end, Nitzberg, Mumford and Shiota had the luminous idea to mimic a natural ability of human vision to complete partially occluded objects, the so-called amodal completion process described and studied by the Gestalt school of psychology and particularly G. Kanizsa [23]. From a series of perceptual experiments, Kanizsa found out that our vision system detects occlusion at a very low level, actually as soon as it detects $T$-junctions, which are points where an object outline abruptly abuts against the outline of another object and forms a junction in the shape of the letter " $T$ ". In particular, our perception of occlusion has nothing to do with a prior recognition of the objects. Having detected the T-junction, our brain performs a continuation of object boundaries between T-junctions (see Figure 1).

As pointed out by Kanizsa, this continuation process relies on many different laws [23] and there is actually no obvious way to model it, even in relatively simple situations [18]. Again, it seems that no process of recognition is involved (see Figure 2). The idea of Mumford, Nitzberg and Shiota was to adapt the theory of Kanizsa to their framework. Given the object boundaries, it is easy to detect T-junctions. Hereafter, the main problem is the completion of object boundaries between T-junctions. As we said, there is no simple model for amodal completion. However, it can be proved that completion curves are in general as short as possible while respecting a principle of good continuation with respect to the edges being completed. Thus, the model proposed by Mumford et al. 


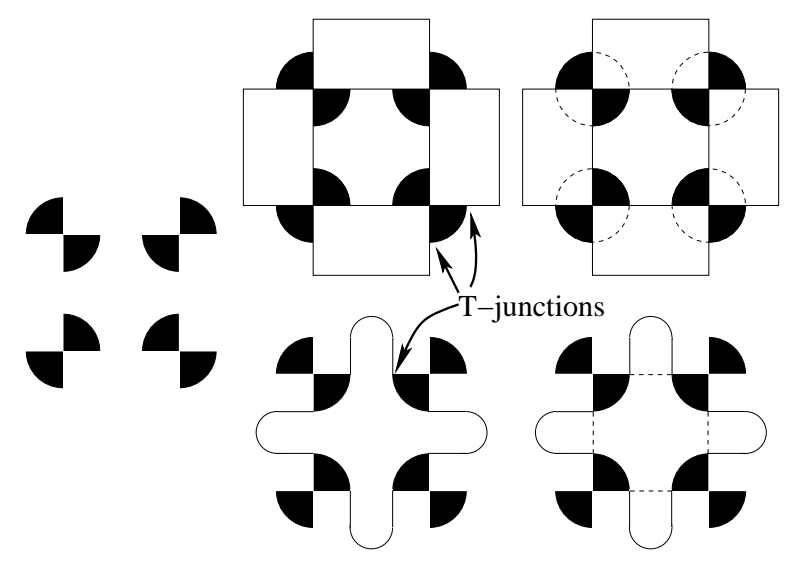

FIG. 1. This example, due to G. Kanizsa [23], illustrates the amodal completion process. Starting from the four objects in the left column, the addition of either four white rectangles or a white cross produces T-junctions (middle column) that conduce our brain to perceive occlusions that, in reality, do not exist. This illustrates perfectly the link between the presence of Tjunctions and the perception of occlusions. Then, our visual system recovers the virtually occluded objects (four black disks in one case and a black square in the other) by connecting T-junctions with completion curves, following a good continuation principle. We have represented those curves with dash lines in the right column.

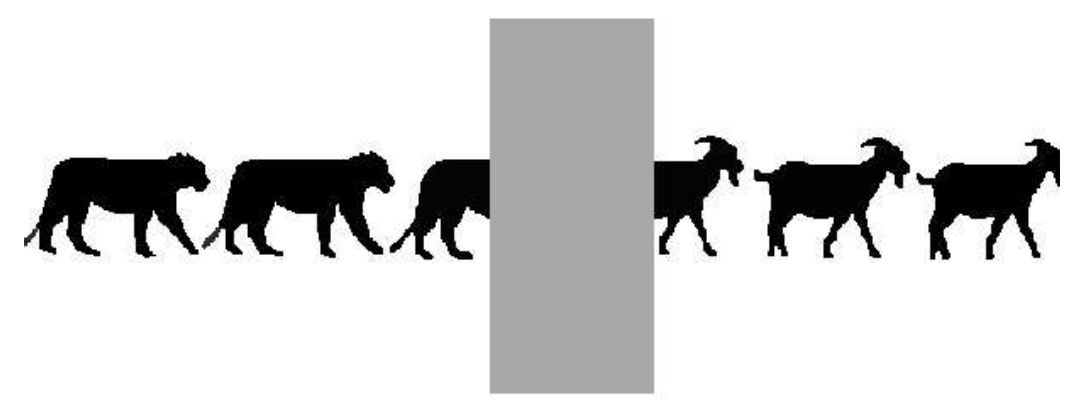

FIG. 2. No recognition process seems to be involved in amodal completion. In this figure (from G. Kanizsa [23]), our brain creates a hybrid animal that obviously contradicts reality.

is the following: given two T-junctions $p_{1}, p_{2}$ and the tangents $t_{1}, t_{2}$ of the respective terminating edges, the continuation curve is the Euler elastica $\Gamma$, that is, the curve minimizing the energy

$$
\int_{\Gamma}\left(\alpha+\beta \kappa^{2}\right) \mathrm{d} \mathcal{H}^{1},
$$

subject to the boundary conditions of beginning at $\left(p_{1}, t_{1}\right)$ and ending at $\left(p_{2}, t_{2}\right)$. Here, $\mathcal{H}^{1}$ denotes the one-dimensional Hausdorff measure, $\kappa$ the curvature on $\Gamma$ and $\alpha, \beta$ are positive reals. Of course, this model is far from being fully satisfactory and, in particular, it does not allow creating corners. However, it sounds reasonable in a first approximation, particularly when the angle between $t_{1}$ and $t_{2}$ is small, and offers a good compromise between shortness and good continuation.

The energy above has in fact a long history. It has been initially studied by Euler [16] in 1744, who investigated the bending of a thin $\operatorname{rod}-\int \kappa^{2} \mathrm{~d} \mathcal{H}^{1}$ is the total bending energy — by forces and 
couples applied at its ends. Then, it was first applied to visual completion by Ullman [41] and Horn [21] and has more recently motivated numerous works (see [24, 37, 39, 40, 43] and the very interesting justification of the model in [30]).

In their paper, Mumford, Nitzberg and Shiota recover partially occluded objects in the following way: among all possible T-junction pairings, the algorithm first disqualifies those for which intensities are too different, based on the underlying reasonable assumption that intensity cannot vary too much along an edge. Then, the algorithm finds the pairings of minimal energy, from which the complete objects can be easily deduced.

Though it was not initially designed for that purpose, it is not difficult to adapt this approach to the problem of recovering missing parts of an image. If $A$ denotes a hole that we want to fill, we can consider $\partial A$ as an edge, compute all T-junctions on $\partial A$ and try to find optimal pairings between them. The result would be a family of overlapping objects given by their boundaries. The grey level at each point of an object $O$ is known only outside $A$ but one can easily imagine a way to define it also on $O \cap A$, e.g. simply putting the average value computed over $O \backslash A$, and this strategy applied to each object would finally give an image where $A$ has been filled.

It is easily seen that the strategy has however a major drawback: its dependence on a prior edge detection process. It is well known indeed that edges are not reliable features in the sense that they cannot be defined in a reliable way. Actually, each edge detector provides a particular definition of edges and, consequently, the image resulting from the strategy above depends as much on the image itself as on the edge detector! In addition, edges furnish a very poor representation of the original image, actually a coarse approximation to the component $u_{1}$ that we defined previously. The image reconstructed with a strategy à la Mumford et al. is therefore rather incomplete since the information outside the missing zone $A$ is not taken into consideration.
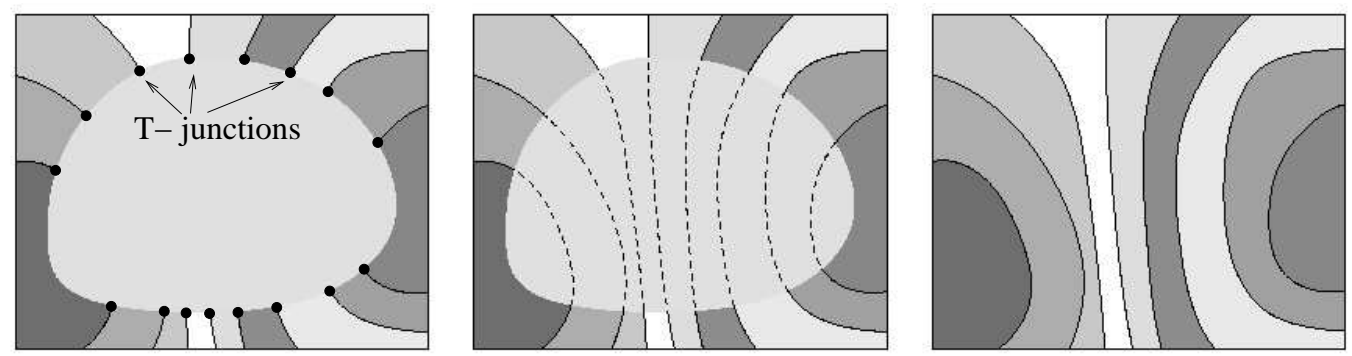

FIG. 3. The algorithm in [28] first detects T-junctions as the intersection points between the occlusion's boundary and the outer level lines (left). Then it computes optimal pairings between compatible T-junctions and draws the corresponding completion curves (middle). Finally, the occlusion is removed by simply filling with the appropriate grey levels (right).

To remedy these drawbacks, it was proposed in [28] (see also [27]) to adapt Mumford et al. strategy to the level lines framework (see Figure 3). Level lines have many advantages in our setting:

- They provide a complete representation of any Borel function $u$ : given the upper level sets $X_{\lambda} u=\{x: u(x) \geqslant \lambda\}$, the image can be easily reconstructed with the formula

$$
u(x)=\sup \left\{\lambda: x \in X_{\lambda} u\right\}
$$

which holds almost everywhere. 
- They are perfectly adapted to the description of image geometry. In particular, the family of level sets is globally invariant with respect to any increasing contrast change, exactly like image objects (the shape of a bird remains the shape of a bird after a contrast change). In contrast, edges are fully contrast-dependent features.

- They are well suited to the BV setting for mainly three reasons:

- almost every level set of a function of bounded variation has finite perimeter (see the next section). In addition to all properties that it implies, the notion of finite perimeter is compatible with a weak notion of connectedness [3] which can be particularly useful for the description of image shapes;

- by the Cavalieri formula, the $\mathrm{L}^{1}$ norm of a measurable function on $\mathbb{R}^{N}$ depends on the $N$-dimensional measure of its level sets;

- by the coarea formula, the total variation of a BV function on $\mathbb{R}^{N}$ depends on the $(N-1)$ dimensional Hausdorff measure of its level lines.

In [28], the authors call disocclusion their method for recovering missing parts of a grey level two-dimensional image, since missing parts can obviously be considered as occlusions "hiding" some information one wants to recover. It is assumed that $u$ is a BV function on the plane known everywhere except on a bounded simply connected open set $A$ with smooth boundary. T-junctions are defined as those points where $\partial A$ intersects the level lines of $u$. The algorithm presented in [28] tries to find optimal pairings between compatible T-junctions, i.e. associated to the same level set, such that the orientation of $D u$ is the same at both points and the optimal associated curve does not cross another completion curve. Both conditions ensure that the new sets obtained by the addition of the completion curves still are level sets. Given two compatible T-junctions $j_{1}$ and $j_{2}$ on $\partial A$ and $\theta_{1}, \theta_{2}$ the corresponding orientations of $D u$ (computed for instance as an average over some neighborhood), the optimal completion curve proposed in [28] is a curve $\Gamma$ that lives in $A$ and minimizes the criterion

$$
\int_{\Gamma}\left(\alpha+\beta|\kappa|^{p}\right) \mathrm{d} \mathcal{H}^{1}+\left(\theta_{1}, n_{1}\right)+\left(\theta_{2}, n_{2}\right) .
$$

Here, $\alpha, \beta$ are positive reals, $p \geqslant 1$ is a real parameter introduced to generalize the elastica energy and the last two terms denote the angles between $\theta_{1}, \theta_{2}$ and the normals to $\Gamma$ at $j_{1}$ and $j_{2}$ respectively. These terms guarantee that, at least in a first approximation, the good continuation principle is satisfied. The global energy to minimize is finally of the form

$$
\int_{-\infty}^{\infty} \sum_{\Gamma \in F_{\lambda}}\left(\int_{\Gamma}\left(\alpha+\beta|\kappa|^{p}\right) \mathrm{d} \mathcal{H}^{1}+\left(\theta_{1}, n_{1}\right)+\left(\theta_{2}, n_{2}\right)\right) \mathrm{d} \lambda,
$$

with $F_{\lambda}$ denoting the family of completion curves associated to the level set $\{u \geqslant \lambda\}$. It must be emphasized that $F_{\lambda}$ is generically finite for almost every $\lambda$, which explains the finite sum [26].

Given an initial BV function outside $A$, the existence of an optimal solution with respect to criterion (2) has been proven in [26] for any $p \geqslant 1$, with the additional assumption in the case $p>1$ that the restriction of $u$ to $\partial A$ takes finitely many values. In contrast to most variational problems, one does not prove directly the existence of an optimal function interpolating the image in $A$ but rather the existence of an optimal family of interpolating level lines from which a function can be recovered.

Recall now that the angle terms were introduced to guarantee the good continuation principle. Another way, more restrictive, to guarantee this principle is to replace the angle constraint with 
a higher order constraint. This can be done in a very logical way by computing the criterion $\int\left(\alpha+\beta|\kappa|^{p}\right) \mathrm{d} \mathcal{H}^{1}$ not only on the completion curve but also on a small piece of the associated level lines outside $A$. If $\tilde{A}$ denotes a set slightly bigger than $A$, our criterion 2 becomes

$$
\int_{-\infty}^{\infty} \sum_{\Gamma \in F_{\lambda}} \int_{\Gamma}\left(\alpha+\beta|\kappa|^{p}\right) \mathrm{d} \mathcal{H}^{1}
$$

where, now, the elements of $F_{\lambda}$ are unions of a completion curve and the restrictions to $\tilde{A} \backslash A$ of the associated level lines. Of course, this criterion makes sense under the assumption that the level lines of the initial BV function are essentially $W^{2, p}$ in $\tilde{A} \backslash A$. In a forthcoming paper [29], the existence of an optimal solution with respect to this new criterion is proved for any $p>1$ without the assumption of finiteness required in [26]. Again, the minimization is performed over a family of curves rather than on a function. Roughly speaking, the existence of optimal curves is proven for a dense family of $\lambda$ using martingale arguments, then a density argument and a series of diagonal extractions give an optimal family of completion curves, from which a solution can be deduced.

Our initial motivation in this paper was precisely to study the disocclusion problem from the viewpoint of the direct method of the calculus of variations. To this end, we first need to rewrite criterion (3) according to a function rather than a family of curves. Assuming for a moment that the curves $\Gamma$ in (3) are the level lines of a smooth function $u$, it is easily seen that the criterion becomes

$$
\int_{-\infty}^{\infty}\left(\int_{\partial\{u \geqslant \lambda\} \cap \tilde{A}}\left(\alpha+\beta|\kappa|^{p}\right) \mathrm{d} \mathcal{H}^{1}\right) \mathrm{d} \lambda .
$$

This criterion can be easily generalized to higher dimensions; if $u$ now denotes a function on $\mathbb{R}^{N}(N \geqslant 2)$, the curvature $\kappa$ can be replaced with the mean curvature vector $\mathbf{H}$ of the hypersurface $\partial\{u \geqslant \lambda\} \cap \tilde{A}$ and the criterion becomes

$$
\int_{-\infty}^{\infty}\left(\int_{\partial\{u \geqslant \lambda\} \cap \tilde{A}}\left(\alpha+\beta|\mathbf{H}|^{p}\right) \mathrm{d} \mathcal{H}^{N-1}\right) \mathrm{d} \lambda,
$$

with $\mathcal{H}^{N-1}$ the $(N-1)$-dimensional Hausdorff measure. Analogously to the two-dimensional case, the minimization of this criterion is equivalent to seeking optimal interpolation hypersurfaces with respect to the energy $\int\left(\alpha+\beta|\mathbf{H}|^{p}\right) \mathrm{d} \mathcal{H}^{N-1}$. Then, it is very easy to formulate the problem according to the function $u$ rather than its level sets by applying equality (7) below, observing that $\nabla u /|\nabla u|$ is orthogonal to the hypersurface $\partial\{u \geqslant \lambda\}$ at every point where $|\nabla u|>0$, and using the change of variables formula. One finally gets the new criterion:

$$
F(u)=\int_{\tilde{A}}|\nabla u|\left(\alpha+\beta\left|\operatorname{div} \frac{\nabla u}{|\nabla u|}\right|^{p}\right) \mathrm{d} x
$$

with the convention that the integrand is 0 wherever $|\nabla u|=0$.

Of course, this criterion makes sense only for a certain class of smooth functions and requires to be relaxed in order to deal with more general functions. As usual in the direct method of the calculus of variations, $F$ is first extended to the whole space $\mathrm{L}^{1}\left(\mathbb{R}^{N}\right)$ and then the relaxed functional associated with $F$ is defined as

$$
\bar{F}(u)=\inf \left\{\liminf _{h \rightarrow \infty} F\left(u_{h}\right): u_{h} \rightarrow u \in \mathrm{L}^{1}\right\}
$$


As we will see in this paper, this relaxed criterion is well adapted to the study of our minimization problem.

Another approach by relaxation, taken by C. Ballester, M. Bertalmio, V. Caselles, G. Sapiro and J. Verdera [6], is based on the functional

$$
G(u, v)=\int_{\tilde{A}}|\operatorname{div} \nu|^{p}(\alpha+\beta|\nabla k \star u|) \mathrm{d} x+\lambda \int_{\tilde{A}}(|\nabla u|-\nabla u \cdot v) \mathrm{d} x
$$

where $v$ is a vector field such that $|v| \leqslant 1$ and $k$ is a smoothing kernel introduced for technical reasons. The advantage of this formulation is the cancellation of the difficulty due to the term $\operatorname{div}(\nabla u /|\nabla u|)$ in $F$. Intuitively, the vector field $v$ plays the role of $\nabla u /|\nabla u|$ but may remain well defined even when $|\nabla u|$ vanishes. The existence of a minimizing couple $(u, v)$ is proved in [6] but it remains unclear whether this approach and ours are equivalent.

We did not mention until now any numerical implementation of the disocclusion model. A practical algorithm for the global minimization of criterion (2) in the case $N=2, p=1$, based on dynamic programming for finding an optimal set of completion curves, has been proposed in [28]. Its performance is illustrated in Figure 4.
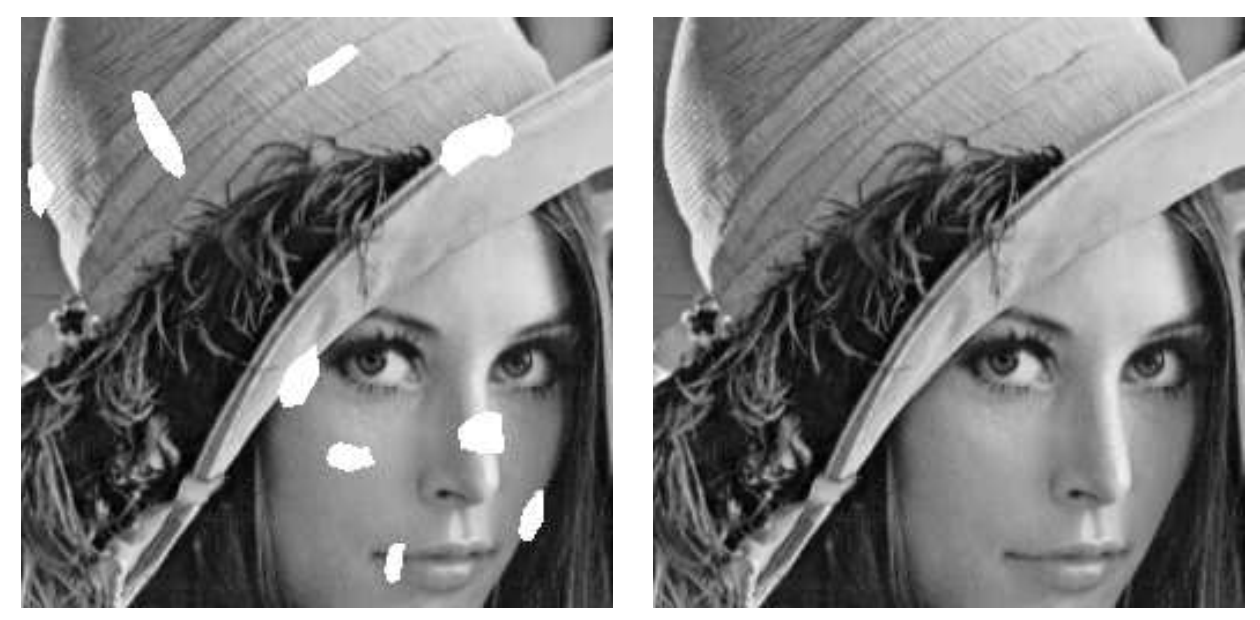

FIG. 4. Left: original image where occlusions are in white. Right: disocclusion performed by the algorithm proposed in [28].

In [10], T. Chan and J. Shen derive the Euler-Lagrange equation associated with criterion (5), in the case $N=2, p>1$. It is a fourth-order equation that raises many problems of unstability and computational time. In addition, the solutions are only local.

Finally, the algorithm proposed in [6] computes local solutions to the minimum problem associated with the functional $G$ defined above. These solutions are obtained through evolutionary equations of order three, thus much handier from the numerical viewpoint than the fourth-order equation in [10]. This approach gives actually very convincing results.

The Euler-Lagrange equation proposed in [10] is obtained through a formal derivation of criterion (5) but is in fact ill-posed. The usual method in such a situation consists in approximating 
$\bar{F}$ by a $\Gamma$-converging family of more regular functionals $F_{\epsilon}$, i.e. a family satisfying:

$$
\begin{array}{ll}
\bar{F}(u) \leqslant \liminf _{\epsilon \rightarrow 0} F_{\epsilon}\left(u_{\epsilon}\right) \quad \text { for every } u_{\epsilon} \rightarrow u & (\Gamma \text {-liminf property }) \\
\exists u_{\epsilon} \rightarrow u \text { such that } \quad \bar{F}(u) \geqslant \limsup _{\epsilon \rightarrow 0} F_{\epsilon}(u) & (\Gamma \text {-lim sup property. })
\end{array}
$$

A crucial fact on $\Gamma$-convergence is that limits of sequences of minimizers of the $F_{\epsilon}$ 's are minimizers of $F$. Thus, the solutions to the well posed Euler-Lagrange equations derived from the functionals $F_{\epsilon}$ can be considered as good approximations of local minimizers of $\bar{F}$, which is particularly interesting from the numerical point of view. Before we introduce the appropriate regular functionals for our problem, let us recall that, in a different context, it has been proven in [17] that an approximation of the solution to

$$
u_{t}=|\nabla u| \operatorname{div}\left(\frac{\nabla u}{|\nabla u|}\right) \quad \text { in }(0, \infty) \times \mathbb{R}^{N}, \quad u(0, \cdot)=u_{0}
$$

is given by the solutions $u^{\epsilon}$ of

$$
u_{t}=\sqrt{\epsilon^{2}+|\nabla u|^{2}} \operatorname{div}\left(\frac{\nabla u}{\sqrt{\epsilon^{2}+|\nabla u|^{2}}}\right)
$$

if the initial function $u_{0}$ is $\mathrm{C}^{1,1}$ and constant at infinity (see [17] for details). To understand better this result, it suffices to remark that if $v:(0, \infty) \times \mathbb{R}^{N+1} \rightarrow \mathbb{R}$ is defined by $v(t, x, z)=u(t, x)+\epsilon z$ then (6) can be rewritten as

$$
v_{t}=|\nabla v| \operatorname{div}\left(\frac{\nabla v}{|\nabla v|}\right)
$$

which coincides with the initial equation.

In the same spirit, let us consider the family of functionals

$$
F_{\epsilon}(u)=\int_{\tilde{A}} \sqrt{|\nabla u|^{2}+\epsilon^{2}}\left(\alpha+\beta\left|\operatorname{div}\left(\frac{\nabla u}{\sqrt{|\nabla u|^{2}+\epsilon^{2}}}\right)\right|^{p}\right) \mathrm{d} x
$$

which take finite values for any $u \in \mathrm{C}^{2}\left(\mathbb{R}^{N}\right)$. Considering $v_{\epsilon}: \mathbb{R}^{N+1} \rightarrow \mathbb{R}$ defined by $v_{\epsilon}(x, z)=$ $u(x)+\epsilon z$, it is easily seen that

$$
\begin{aligned}
F_{\epsilon}(u) & =\int_{\tilde{A}}\left|\nabla v_{\epsilon}\right|\left(\alpha+\beta\left|\operatorname{div}\left(\frac{\nabla v_{\epsilon}}{\left|\nabla v_{\epsilon}\right|}\right)\right|^{p}\right) \mathrm{d} x \\
& =\int_{\tilde{A}} \int_{-1 / 2}^{1 / 2}\left|\nabla v_{\epsilon}\right|\left(\alpha+\beta\left|\operatorname{div}\left(\frac{\nabla v_{\epsilon}}{\left|\nabla v_{\epsilon}\right|}\right)\right|^{p}\right) \mathrm{d} x \mathrm{~d} z
\end{aligned}
$$

which is exactly the $(N+1)$-dimensional version of $F$. This observation combined with Theorem 6 in Section 4 shows that the $\Gamma$-liminf property is satisfied by $\bar{F}$ and the family $\left(F_{\epsilon}\right)_{\epsilon>0}$. We were unfortunately unable to prove more than that and can only state the following

Conjecture For $\epsilon>0$, let $F_{\epsilon}: \mathrm{L}^{1}\left(\mathbb{R}^{N}\right) \rightarrow[0, \infty]$ be defined by

$$
F_{\epsilon}(u)= \begin{cases}\int_{\tilde{A}} \sqrt{|\nabla u|^{2}+\epsilon^{2}}\left(\alpha+\beta\left|\operatorname{div} \frac{\nabla u}{\sqrt{|\nabla u|^{2}+\epsilon^{2}}}\right|^{p}\right) \mathrm{d} x & \text { if } u \in \mathrm{C}_{\mathrm{c}}^{2}\left(\mathbb{R}^{N}\right), \\ & \text { otherwise. }\end{cases}
$$


Then, for $N<p<\infty$ and for every $\left(\epsilon_{h}\right)_{h \in \mathbb{N}} \rightarrow 0$,

$$
\bar{F}=\Gamma-\lim _{h \rightarrow \infty} F_{\epsilon_{h}} .
$$

This paper is organized as follows. Section 2 introduces some notations and basic facts about tools from geometric measure theory that we shall need. In Section 3, we prove a locality result for the mean curvature vector $\mathbf{H}$ of integral $(N-1)$-varifolds when $\mathbf{H} \in \mathrm{L}^{p}, p>N-1, p \geqslant 2$ (Theorem 2). A direct consequence of this result is the lower semicontinuity (Theorem 4) of the functional

$$
\int_{\partial E}\left(1+\left|\mathbf{H}_{E}\right|^{p}\right) \mathrm{d} \mathcal{H}^{N-1}, \quad p>N-1, N \geqslant 3,
$$

with respect to convergence in $\mathrm{L}^{1}$ in the class of sets $E \subset \mathbb{R}^{N}$ with $\partial E \in \mathrm{C}^{2}$. This result extends to higher dimensions a previous result due to G. Bellettini, G. Dal Maso and M. Paolini [7] for $N=2$, $p>1$.

Section 4 is devoted to the study of the disocclusion problem in dimension $N$. We prove the existence of an optimal solution (Theorem 5 , the coincidence between $F$ and the associated relaxed functional $\bar{F}$ for smooth functions (Theorem 6 ) and give some results on the regularity of the optimal solution in the particular case $N=2$ (Corollary 1). For the sake of simplicity and with absolutely no loss of generality, we shall assume in what follows that $\alpha=\beta=1$.

\section{Notations and main facts about varifolds}

We collect below, for the reader's convenience, the main facts about varifolds (see for instance [4, 19, 38]).

We let $\mathcal{L}\left(\mathbb{R}^{n+k}\right)$ denote the space of linear maps from $\mathbb{R}^{n+k}$ onto itself, equipped with the usual scalar product $A \bullet B=\operatorname{trace}\left(A^{*} B\right) . G(n+k, n)$ denotes the space of $n$-dimensional unoriented subspaces of $\mathbb{R}^{n+k}$ and we shall often identify an $n$-subspace $S \in G(n+k, n)$ with the associated orthogonal projection $p_{S} \in \mathcal{L}\left(\mathbb{R}^{n+k}\right)$ given by the matrix $p_{S}^{i j}=e_{i} \cdot p_{S}\left(e_{j}\right)$ with respect to the standard orthonormal basis $e_{1}, \ldots, e_{n+k}$ for $\mathbb{R}^{n+k} . G(n+k, n)$ is equipped with the metric

$$
\left\|p_{S}-p_{T}\right\|:=\left(\sum_{i, j=1}^{n+k}\left(p_{S}^{i j}-p_{T}^{i j}\right)^{2}\right)^{1 / 2}
$$

induced by the scalar product $\bullet$ on $\mathcal{L}\left(\mathbb{R}^{n+k}\right)$. The tensor product $v \otimes w$ of two vectors $v, w \in \mathbb{R}^{n+k}$ is in $\mathcal{L}\left(\mathbb{R}^{n+k}\right)$ and satisfies, for any $S \in G(n+k, n)$,

$$
v \otimes w \bullet S=S(v) \bullet w=v \bullet S(w)=S(v) \bullet S(w) .
$$

For a subset $A \subset \mathbb{R}^{n+k}$ we define the Grassmannian

$$
G_{n}(A)=A \times G(n+k, n)
$$

equipped with the product metric. By an $n$-varifold on an open subset $U$ of $\mathbb{R}^{n+k}$ we mean any Radon measure $V$ on $G_{n}(U)$. It is associated with a Radon measure $\mu_{V}$ on $U$ (called the weight of $V$ ) defined by

$$
\mu_{V}(A)=V\left(\pi^{-1}(A)\right), \quad A \subset U \text { Borel, }
$$

where $\pi$ is the projection $(x, S) \mapsto x$ of $G_{n}(U)$ onto $U$. 
Given $M$, a countably $\mathcal{H}^{n}$-rectifiable subset of $\mathbb{R}^{n+k}$, and $\theta$, a positive and locally $\mathcal{H}^{n}$-integrable function on $M$, we define the associated $n$-rectifiable varifold $V \equiv \underline{\underline{\mathbf{v}}}(M, \theta)$ by

$$
V(A)=\mu_{V}(\pi(T M \cap A)), \quad A \subset G_{n}(U) \text { Borel, }
$$

where $\mu_{V}:=\mathcal{H}^{n}\left\llcorner\theta\right.$ is the weight of $V, T M=\left\{\left(x, T_{x} M\right): x \in M^{*}\right\}$ and $M^{*}$ stands for the set of all $x \in M$ such that $M$ has an approximate tangent space $T_{x} M$ with respect to $\theta$ at $x$, i.e.

$$
\lim _{\lambda \downarrow 0} \lambda^{-n} \int_{M} f\left(\lambda^{-1}(z-x)\right) \theta(z) \mathrm{d} \mathcal{H}^{n}(z)=\theta(x) \int_{T_{x} M} f(y) \mathrm{d} \mathcal{H}^{n}(y) \quad \forall f \in \mathrm{C}_{\mathrm{c}}^{0}\left(\mathbb{R}^{n+k}\right) .
$$

We say that $V=\underline{\underline{\mathbf{v}}}(M, \theta)$ is an integral varifold if the function $\theta$ is integer-valued. We remark that $\mathcal{H}^{n}\left(M \backslash M^{*}\right) \stackrel{=}{=} 0$ and that the approximate tangent spaces of $M$ with respect to two different positive $\mathcal{H}^{n}$-integrable functions $\theta, \tilde{\theta}$ coincide $\mathcal{H}^{n}$-a.e. in $M$.

The first variation of the $n$-varifold $V$, denoted by $\delta V$, is the linear functional on $\mathrm{C}_{\mathrm{c}}^{1}\left(U, \mathbb{R}^{n+k}\right)$ defined by

$$
\delta V(X):=\int_{G_{n}(U)} \operatorname{div}_{S} X \mathrm{~d} V(x, S)
$$

where, for any $S \in G(n+k, n)$,

$$
\operatorname{div}_{S} X:=\sum_{i=1}^{n+k} \nabla_{i}^{S} X^{i}=\sum_{i=1}^{n}\left\langle\tau_{i}, D_{\tau_{i}} X\right\rangle
$$

where $\tau_{1}, \ldots, \tau_{n}$ is an orthonormal basis for $S$ and $\nabla_{i}^{S}=e_{i} . \nabla^{S}$ with $\nabla^{S} f(x)=S(\nabla f(x)), f \in$ $\mathrm{C}^{1}(U)$.

A varifold $V$ is said to have locally bounded first variation in $U$ if for each $W \subset \subset U$ there is a constant $c<\infty$ such that $|\delta V(x)| \leqslant c \sup _{U}|X|$ for any $X \in \mathrm{C}_{\mathrm{c}}^{1}\left(U, \mathbb{R}^{n+k}\right)$ with spt $|X| \subset W$. By the Riesz representation theorem, it follows that there exist a Radon measure $\|\delta V\|$ on $U$ - the total variation measure of $\delta V$-and a $\|\delta V\|$-measurable function $v$ with $|v|=1\|\delta V\|$-a.e. in $U$ satisfying

$$
\delta V(X)=-\int_{U} v \cdot X \mathrm{~d}\|\delta V\| \quad \forall X \in \mathrm{C}_{\mathrm{c}}^{1}\left(U, \mathbb{R}^{n+k}\right) .
$$

A varifold $V$ is said to have mean curvature in $\mathrm{L}^{p}$ if $\|\delta V\|$ is absolutely continuous with respect to $\mu_{V}$ and its density belongs to $L^{p}$. The density will be denoted by $\mathbf{H}_{V}$ and it will be called the generalized mean curvature of $V$.

In the case when $M$ is a smooth $n$-dimensional submanifold of $\mathbb{R}^{n+k}$ with $\bar{M} \backslash M \cap U=\emptyset$, the divergence theorem on manifolds implies that the generalized mean curvature of the varifold $\underline{\underline{\mathbf{v}}}(M, 1)$ is exactly the classical mean curvature of $M$. When $k=1$ (i.e. codimension 1 manifolds) the mean curvature vector can be locally computed by

$$
\mathbf{H}=-\operatorname{div}(v) v
$$

where $v$ is a unit vector field orthogonal to $M$. 


\section{Locality of the mean curvature and applications}

The main result of this section is stated in Theorem 2, whose proof is based on the quadratic decay of the tilt-excess established by R. Schätzle in [36]. We recall his result below.

THEOREM 1 (Quadratic tilt-excess decay [36, Thm. 5.1]) Let $U=\mathbf{v}(M, \theta)$ be an integral $(N-1)$ varifold in an open set $\Omega \subseteq \mathbb{R}^{N}$ with $\mathbf{H}_{U} \in \mathrm{L}_{\mathrm{loc}}^{p}\left(\mu_{U}\right), p>N-1, p \geqslant 2$. Then for $\mu_{U}$-almost all $x \in \operatorname{spt} \mu_{U}$, the tilt-excess

$$
\operatorname{tiltex}_{\mu}(x, \rho):=\rho^{1-N} \int_{B_{\rho}^{N}(x)}\left\|T_{y} M-T_{x} M\right\|^{2} \mathrm{~d} \mu_{U}(y)
$$

decays quadratically, that is,

$$
\operatorname{tiltex}_{\mu}(x, \rho)=O_{x}\left(\rho^{2}\right)
$$

THEOREM 2 (Locality of the mean curvature) Let $U=\mathbf{v}\left(M, \theta_{U}\right), V=\mathbf{v}\left(M^{\prime}, \theta_{V}\right)$ be integral $(N-1)$-varifolds in $\Omega \subseteq \mathbb{R}^{N}$. If $\mathbf{H}_{U} \in \mathrm{L}_{\text {loc }}^{p}\left(\mu_{U}\right)$ and $\overline{\overline{\mathbf{H}}}_{V} \in \mathrm{L}_{\text {loc }}^{p}\left(\mu_{V}\right)$ for some $p>N-1$, $p \geqslant 2$ then

$$
\mathbf{H}_{U}(x)=\mathbf{H}_{V}(x)
$$

for $\mathcal{H}^{N-1}$-almost all $x \in M \cap M^{\prime}$.

Proof. Given nonzero integers $\theta_{0}, \theta_{1}$, we call $x \in M \cap M^{\prime}$ a generic point of order $\left(\theta_{0}, \theta_{1}\right)$ if

(i) $\theta^{N-1}\left(M \cap M^{\prime}, x\right)=1$ and $\theta_{0}=\theta^{N-1}\left(\mu_{U}, x\right), \theta_{1}=\theta^{N-1}\left(\mu_{V}, x\right)$;

(ii) $x$ is a Lebesgue point of $\mathbf{H}_{U}$ and $\mathbf{H}_{V}$;

(iii) $\mu_{U}, \mathbf{H}_{U} \mu_{U}, \mu_{V}$ and $\mathbf{H}_{V} \mu_{V}$ have the same approximate tangent plane at $x$ (with multiplicities $\theta_{0}, \theta_{0} \mathbf{H}_{U}(x), \theta_{1}$ and $\theta_{1} \mathbf{H}_{V}(x)$ respectively) which in turn coincide with the approximate tangent plane $T=T_{x} M=T_{x} M^{\prime}$

(iv) $\mathbf{H}_{U}(x)$ and $\mathbf{H}_{V}(x)$ are orthogonal to $T$.

The theory of rectifiable sets and of rectifiable measures (see for instance [4,38]) ensures that $\mathcal{H}^{N-1}$-almost all points in $x \in M \cap M^{\prime}$ have properties (i), (ii), (iii). The proof that also condition (iv) holds $\mathcal{H}^{N-1}$-a.e. is much harder (see [8, Thm. 5.8]). Therefore $\mathcal{H}^{N-1}$-almost every point of $M \cap M^{\prime}$ is generic of order $\left(\theta_{0}, \theta_{1}\right)$ for some $\theta_{0}, \theta_{1}$. We fix $\theta_{0}, \theta_{1}$ and a generic point $x$ of the corresponding order.

Following the proof of Lemma 6.3 in [35], we choose $\chi \in \mathrm{C}_{0}^{\infty}\left(B_{1}^{N}(0)\right)$ rotationally symmetric with $0 \leqslant \chi \leqslant 1$ and $\chi \equiv 1$ on $B_{1 / 2}^{N}(0)$. Setting $\chi_{r}(y):=\chi\left(r^{-1}(y-x)\right)$, using (iii) we have

$$
\lim _{r \rightarrow 0^{+}} r^{1-N} \delta U\left(\chi_{r}\right)=-\lim _{r \rightarrow 0^{+}} r^{1-N} \int_{B_{r}^{N}(x)} \chi_{r} \mathbf{H}_{U} \mathrm{~d} \mu_{U}=\theta_{0} \mathbf{H}_{U}(x) \int_{T_{x} M \cap B_{1}^{N}(0)} \chi \mathrm{d} \mathcal{H}^{N-1},
$$

and

$$
\lim _{r \rightarrow 0^{+}} r^{1-N} \delta V\left(\chi_{r}\right)=-\lim _{r \rightarrow 0^{+}} r^{1-N} \int_{B_{r}^{N}(x)} \chi_{r} \mathbf{H}_{V} \mathrm{~d} \mu_{V}=\theta_{1} \mathbf{H}_{V}(x) \int_{T_{x} M^{\prime} \cap B_{1}^{N}(0)} \chi \mathrm{d} \mathcal{H}^{N-1}
$$

Now we choose $v(x)$ normal to $T_{x} M=T_{x} M^{\prime}$ and we deduce by (iv) that $\mathbf{H}_{U}(x), \mathbf{H}_{V}(x) \in$ $\operatorname{span}\{v(x)\}$. Hence, in order to show that they actually coincide, it suffices to prove that

$$
\lim _{r \rightarrow 0^{+}} r^{1-N}\left[\theta_{1} \delta U\left(\chi_{r}\right)-\theta_{0} \delta V\left(\chi_{r}\right)\right] v(x)=0 .
$$


Let us now denote by $A$ the collection of all generic points of order $\left(\theta_{0}, \theta_{1}\right)$. We assume, in addition, that

(v) $\lim _{r \rightarrow 0^{+}} r^{1-N} \mathcal{H}^{N-1}\left(B_{r}^{N}(x) \backslash A\right)=0$.

It is a consequence of Theorem 2.9.11 in [19] that $\mathcal{H}^{N-1}$-almost every generic point of order $\left(\theta_{0}, \theta_{1}\right)$ has this property. Then, we notice for $W=U$ or $W=V$ that

$$
\int_{B_{r}^{N}(x)} \chi_{r} \mathbf{H}_{W} \mathrm{~d} \mu_{W}=-\int_{B_{r}^{N}(x)} T_{y} W\left(D \chi_{r}\right) \mathrm{d} \mu_{W}(y),
$$

thus

$$
\begin{aligned}
{\left[\int_{B_{r}^{N}(x)} \chi_{r} \theta_{1} \mathbf{H}_{U} \mathrm{~d} \mu_{U}\right.} & \left.-\int_{B_{r}^{N}(x)} \chi_{r} \theta_{0} \mathbf{H}_{V} \mathrm{~d} \mu_{V}\right] \\
& =-\left[\int_{B_{r}^{N}(x)} T_{y} M\left(D \chi_{r}\right) \theta_{1} \mathrm{~d} \mu_{U}(y)-\int_{B_{r}^{N}(x)} T_{y} M^{\prime}\left(D \chi_{r}\right) \theta_{0} \mathrm{~d} \mu_{V}(y)\right] .
\end{aligned}
$$

Then, by looking at the $v(x)$ component, we get

$$
\begin{aligned}
& {\left[\int_{B_{r}^{N}(x)} \chi_{r} \theta_{1} \mathbf{H}_{U} \mathrm{~d} \mu_{U}-\int_{B_{r}^{N}(x)} \chi_{r} \theta_{0} \mathbf{H}_{V} \mathrm{~d} \mu_{V}\right] \cdot v(x)} \\
& \quad=-\left[\int_{B_{r}^{N}(x)}\left(D \chi_{r}(y) \otimes v(x)\right) T_{y} M \theta_{1} \mathrm{~d} \mu_{U}(y)-\int_{B_{r}^{N}(x)}\left(D \chi_{r}(y) \otimes v(x)\right) T_{y} M^{\prime} \theta_{0} \mathrm{~d} \mu_{V}(y)\right] .
\end{aligned}
$$

Now we use the fact that $v(x)$ is normal to $T$, that $T_{y} M=T_{y} M^{\prime}, \theta_{U}(y)=\theta_{0}$ and $\theta_{V}(y)=\theta_{1}$ on $A$ to obtain

$$
\begin{aligned}
{\left[\int_{B_{r}^{N}(x)} \chi_{r} \theta_{1} \mathbf{H}_{U} \mathrm{~d} \mu_{U}-\int_{B_{r}^{N}(x)} \chi_{r} \theta_{0} \mathbf{H}_{V} \mathrm{~d} \mu_{V}\right] \cdot v(x) } \\
=-\left[\int_{B_{r}^{N}(x)}\left(D \chi_{r}(y) \otimes v(x)\right)\left(T_{y} M-T\right) \theta_{1} \mathrm{~d} \mu_{U}(y)\right. \\
\left.-\int_{B_{r}^{N}(x)}\left(D \chi_{r}(y) \otimes v(x)\right)\left(T_{y} M^{\prime}-T\right) \theta_{0} \mathrm{~d} \mu_{V}(y)\right] \\
=-\left[\theta_{1} \int_{B_{r}^{N}(x) \backslash A}\left(D \chi_{r}(y) \otimes v(x)\right)\left(T_{y} M-T\right) \mathrm{d} \mu_{U}(y)\right. \\
\left.-\theta_{0} \int_{B_{r}^{N}(x) \backslash A}\left(D \chi_{r}(y) \otimes v(x)\right)\left(T_{y} M^{\prime}-T\right) \mathrm{d} \mu_{V}(y)\right] .
\end{aligned}
$$

Setting

$$
R_{r, W}=r^{1-N} \int_{B_{r}^{N}(x) \backslash A}\left(D \chi_{r}(y) \otimes v(x)\right)\left(T_{y} W-T\right) \mathrm{d} \mu_{W}(y)
$$

for $W=U, V$ we obtain

$$
\lim _{r \rightarrow 0^{+}} r^{1-N}\left[\theta_{1} \delta U\left(\chi_{r}\right)-\theta_{0} \delta V\left(\chi_{r}\right)\right] v(x)=\lim _{r \rightarrow 0^{+}}\left[\theta_{1} R_{r, U}-\theta_{0} R_{r, V}\right] .
$$


Then we estimate, for $W=U, V$,

$$
\begin{aligned}
\left|R_{r, W}\right| & \leqslant C_{\chi} r^{-N} \int_{B_{r}^{N}(x) \backslash A}\left\|T_{y} W-T\right\| \mathrm{d} \mu_{W}(y) \\
& \leqslant C_{\chi}\left(r^{1-N} \mu_{W}\left(B_{r}^{N}(x) \backslash A\right)\right)^{1 / 2}\left(r^{-1-N} \int_{B_{r}^{N}(x)}\left\|T_{y} W-T\right\|^{2} \mathrm{~d} \mu_{W}(y)\right)^{1 / 2},
\end{aligned}
$$

where $C_{\chi}$ is such that $\sup _{B_{1}^{N}(0)}|D \chi| \leqslant C_{\chi}$. By conditions (iv), (v) and by Theorem 1 the first factor is infinitesimal as $r \rightarrow 0^{+}$and the second factor is bounded, hence 8 holds and the proof is complete.

Theorem 2 is a key point in the proof of the lower semicontinuity of the mean curvature's $\mathrm{L}^{p}$ norm, stated in Theorem 4 below, which is a generalization to higher dimensions of a previous result obtained in dimension two by G. Bellettini, G. Dal Maso and M. Paolini in [7]. We recall their result below.

THEOREM 3 ([7, Thm. 7.1]) Let $\Omega$ be an open subset of $\mathbb{R}^{2}$, let $p>1$ and let $E$ be an open bounded subset of $\mathbb{R}^{2}$ such that $\partial E \cap \Omega \in \mathrm{C}^{2}$. Then

$$
\int_{\partial E \cap \Omega}\left(1+|\kappa|^{p}\right) \mathrm{d} \mathcal{H}^{1} \leqslant \liminf _{h \rightarrow \infty} \int_{\partial E_{h} \cap \Omega}\left(1+\left|\kappa_{h}\right|^{p}\right) \mathrm{d} \mathcal{H}^{1}
$$

for any sequence $\left(E_{h}\right)_{h \in \mathbb{N}}$ of bounded open sets such that $\partial E_{h} \cap \Omega \in \mathrm{C}^{2}$ and $E_{h} \rightarrow E$ in $\mathrm{L}^{1}(\Omega)$ as $h \rightarrow \infty$.

THEOREM 4 (Lower semicontinuity of the mean curvature's $\mathrm{L}^{p}$ norm) Let $\Omega$ be an open subset of $\mathbb{R}^{N}, N \geqslant 3$, and $p>N-1$. Let $\left\{E_{h}\right\}_{h \in \mathbb{N}} \subset \mathbb{R}^{N}$ converge in $\mathrm{L}^{1}(\Omega)$ to $E$, with $\partial E_{h} \cap \Omega \in \mathrm{C}^{2}$ and $\partial E \cap \Omega \in \mathrm{C}^{2}$. Then

$$
\int_{\partial E \cap \Omega}\left(1+\left|\mathbf{H}_{E}\right|^{p}\right) \mathrm{d} \mathcal{H}^{N-1} \leqslant \liminf _{h \rightarrow \infty} \int_{\partial E_{h} \cap \Omega}\left(1+\left|\mathbf{H}_{E_{h}}\right|^{p}\right) \mathrm{d} \mathcal{H}^{N-1},
$$

where $\mathbf{H}_{E}$ (resp. $\mathbf{H}_{E_{h}}$ ) denotes the mean curvature vector on $\partial E \cap \Omega$ (resp. $\partial E_{h} \cap \Omega$ ).

Proof. Due to the lower semicontinuity of the perimeter, it is clearly enough to prove the part of the claim that involves curvature. We can assume that the right hand side of the above inequality is finite, otherwise the result is trivial. In addition, possibly taking a subsequence, there is no loss of generality if we assume that

$$
\sup _{h \in \mathbb{N}} \int_{\partial E_{h} \cap \Omega}\left(1+\left|\mathbf{H}_{E_{h}}\right|^{p}\right) \mathrm{d} \mathcal{H}^{N-1} \leqslant C<\infty .
$$

Let $V_{h}=\mathbf{v}\left(\partial E_{h} \cap \Omega, 1\right)$ be the unit-density rectifiable $(N-1)$-varifolds associated with the sets $E_{h} \cap \Omega$ and let $\mu_{V_{h}}=\mathcal{H}^{N-1}\left\llcorner\partial E_{h} \cap \Omega\right.$ be the corresponding weights. By the divergence theorem, the first variation of the $V_{h}$ 's in $\Omega$ can be written as

$$
\delta V_{h}(X)=-\int_{\Omega} X \cdot \mathbf{H}_{E_{h}} \mathrm{~d} \mu_{V_{h}} \quad \forall X \in \mathrm{C}_{\mathrm{c}}^{1}\left(\Omega, \mathbb{R}^{N}\right),
$$

hence the $\mathrm{L}^{p}$ norms of $\delta V_{h}$ with respect to $\mu_{V_{h}}$ are uniformly bounded. 
By Allard's compactness theorem (see [1] or Theorem 42.7 in [38]) we find, possibly passing to a subsequence, that there exists a limit integral $(N-1)$-varifold $V$ in $\Omega$ such that $V_{h} \rightarrow V$ and $V=\mathbf{v}\left(M, \theta_{V}\right)$ with $M$ a countably $\mathcal{H}^{N-1}$-rectifiable set and $\theta_{V}$ a positive integer-valued and locally $\mathcal{H}^{N-1}$-integrable function on $\Omega$. As $\delta V_{h}=\mathbf{H}_{E_{h}} \mathrm{~d} \mu_{V_{h}} \rightarrow \delta V$, a well-known lower semicontinuity theorem (see for instance Example 2.36 in [4]) implies that $\delta V=\mathbf{H}_{V} \mu_{V}$ with $\mathbf{H}_{V} \in L^{p}\left(\mu_{V}\right)$ and

$$
\int_{M} \theta_{V}\left|\mathbf{H}_{V}\right|^{p} \mathrm{~d} \mathcal{H}^{N-1} \leqslant \liminf _{h \rightarrow \infty} \int_{\partial E_{h} \cap \Omega}\left|\mathbf{H}_{E_{h}}\right|^{p} \mathrm{~d} \mathcal{H}^{N-1} .
$$

Notice that so far we used only the fact that $p>1$.

Now we show that $\mathcal{H}^{N-1}$-almost all points in $\partial E \cap \Omega$ belong to $M$ and $\mathbf{H}_{V}$ coincides with the classical mean curvature $\mathbf{H}_{E}$ for $\mathcal{H}^{N-1}$-almost every point of $\partial E \cap \Omega$ whenever $p>N-1$.

Let $x \in \partial E \cap \Omega$. Since $E$ has finite perimeter, for all $r>0$ except possibly for a countable set, $\mu_{V}\left(\partial B_{r}^{N}(x)\right)=0$, hence

$\mu_{V}\left(B_{r}^{N}(x)\right)=\lim _{h \rightarrow \infty} \mu_{V_{h}}\left(B_{r}^{N}(x)\right)=\lim _{h \rightarrow \infty} \mathcal{H}^{N-1}\left(B_{r}^{N}(x) \cap \partial E_{h} \cap \Omega\right) \geqslant \mathcal{H}^{N-1}\left(B_{r}^{N}(x) \cap \partial E \cap \Omega\right)$,

by the lower semicontinuity of the perimeter. It follows that $x$ is a point where the lower $(N-1)$ dimensional density of $\mu_{V}$ is strictly positive. As $\mu_{V}=\theta_{V} \mathcal{H}^{N-1}\left\llcorner M, \mathcal{H}^{N-1}\right.$-almost every point with this property belongs to $M$.

Let $U:=\mathbf{v}(\partial E \cap \Omega, 1)$ be the unit-density rectifiable $(N-1)$-varifold in $\Omega$ associated with $\partial E \cap \Omega$. By the divergence theorem we have

$$
\mathbf{H}_{U}(x)=\mathbf{H}_{E}(x)
$$

for $\mathcal{H}^{N-1}$-almost all $x \in \partial E \cap \Omega$. Since $\partial E \cap \Omega \in \mathrm{C}^{2}$, it is easily seen that $\mathbf{H}_{U} \in \mathrm{L}_{\mathrm{loc}}^{p}\left(\mu_{U}\right)$. By Theorem 2 we obtain

$$
\mathbf{H}_{E}(x)=\mathbf{H}_{U}(x)=\mathbf{H}_{V}(x)
$$

for $\mathcal{H}^{N-1}$-almost all $x \in M \cap \partial E \cap \Omega$ and therefore for $\mathcal{H}^{N-1}$-almost all $x \in \partial E \cap \Omega$.

Plugging this into 10 and using $\theta_{V}(x) \geqslant 1$ for $\mathcal{H}^{N-1}$-almost every $x \in M$, we finally obtain

$$
\int_{\partial E \cap \Omega}\left|\mathbf{H}_{E}\right|^{p} \mathrm{~d} \mathcal{H}^{N-1} \leqslant \int_{\partial E \cap \Omega}\left|\mathbf{H}_{V}\right|^{p} \theta_{V} \mathrm{~d} \mathcal{H}^{N-1} \leqslant \liminf _{h \rightarrow \infty} \int_{\partial E_{h}}\left|\mathbf{H}_{E_{h}}\right|^{p} \mathrm{~d} \mathcal{H}^{N-1}
$$

and the theorem follows.

REMARK 1 The varifold arguments we use require the technical assumption $p \geqslant 2$, which prevents the result by Bellettini et al. from being a particular case of Theorem 4 whenever $N=2$ and $1<p<2$.

REMARK 2 Whenever $\mathcal{H}^{N-1}(\partial E \cap \Omega)=\lim _{h \rightarrow \infty} \mathcal{H}^{N-1}\left(\partial E_{h} \cap \Omega\right)$, the lower semicontinuity is true for any $p \geqslant 1$. This is an easy consequence of (10) and of Reshetnyak's continuity theorem (see for instance [4, Thm. 2.39]), which implies that $V \equiv \mathbf{v}(\partial E \cap \Omega, 1)$.

\section{Analysis of the disocclusion problem}

Let $\Omega$ be a bounded open set in $\mathbb{R}^{N}(N \geqslant 2)$ with Lipschitz boundary, representing the image domain, let $A \subset \subset \Omega$ be an open, connected set with Lipschitz boundary representing the occlusion 
and let $u_{0} \in \mathrm{BV}(\Omega \backslash \bar{A})$ be the original image. By Theorem 3.87 in [4], for any $\lambda \in \mathbb{R}$, the function given by $\left.u\right|_{\Omega \backslash \bar{A}}=u_{0}$ and $\left.u\right|_{A}=\lambda$ belongs to $\operatorname{BV}(\Omega)$. This ensures that the occlusion can always be filled in.

Let $\mathcal{O}(\Omega)$ denote the family of open subsets of $\Omega$. We consider the functional mapping $\mathrm{L}^{1}(\Omega) \times$ $\mathcal{O}(\Omega)$ onto $[0, \infty]$ and defined for every $(u, B) \in \mathrm{L}^{1}(\Omega) \times \mathcal{O}(\Omega)$ by

$$
F_{p}(u, B):= \begin{cases}\int_{B}|\nabla u|\left(1+\left|\operatorname{div} \frac{\nabla u}{|\nabla u|}\right|^{p}\right) \mathrm{d} x & \text { if } u \in \mathrm{C}^{2}(B), \\ & \text { otherwise, }\end{cases}
$$

with the convention that the integrand is 0 wherever $|\nabla u|=0$.

The relaxed functional associated with $F$ is defined for every $(u, B) \in \mathrm{L}^{1}(\Omega) \times \mathcal{O}(\Omega)$ by

$$
\overline{F_{p}}(u, B):=\inf \left\{\liminf _{h \rightarrow \infty} F_{p}\left(u_{h}, B\right): u_{h} \stackrel{\mathrm{L}^{1}(B)}{\longrightarrow} u\right\} .
$$

Since $F_{p}(u, B) \geqslant \int_{B}|\nabla u| \mathrm{d} x$ whenever $u \in \mathrm{C}^{2}(B)$ the lower semicontinuity of the total variation yields

$$
\overline{F_{p}}(u, B) \geqslant|D u|(B) \quad \forall(u, B) \in \mathrm{L}^{1}(\Omega) \times \mathcal{O}(\Omega) .
$$

In the following we assume that there exist an open set $\tilde{A} \subset \Omega$ such that $\tilde{A} \supset \mathcal{A}$ and a function $u \in \mathrm{L}^{1}(\Omega)$ such that $u=u_{0}$ on $\Omega \backslash \bar{A}$ and $\overline{F_{p}}(u, \tilde{A})<\infty$. This could be considered as a mild regularity and compatibility condition between the image and the occlusion.

THEOREM 5 The problem

$$
\operatorname{Min}\left\{\overline{F_{p}}(u, \tilde{A}): u=u_{0} \text { on } \Omega \backslash \bar{A}\right\}
$$

has at least one solution $u \in \mathrm{BV}(\Omega)$.

Proof. Let $\left(v_{h}\right)_{h \in \mathbb{N}} \subset \mathrm{L}^{1}(\Omega)$ be a minimizing sequence. Without loss of generality we may assume that $\sup _{h \in \mathbb{N}} \overline{F_{p}}\left(v_{h}, \tilde{A}\right)<\infty$. Then 11 yields $\sup _{h \in \mathbb{N}}\left|D v_{h}\right|(\tilde{A})<\infty$, and therefore $\sup _{h \in \mathbb{N}}\left|D v_{h}\right|(\Omega)<\infty$ because $v_{h}=u_{0}$ on $\bar{\Omega} \backslash \bar{A}$. Since the values of $v_{h}$ are fixed on $\Omega \backslash \bar{A}$, the generalized Poincaré inequality in Theorem 5.11.1 of [45] shows that the $\mathrm{L}^{1}(\Omega)$ norms of $v_{h}$ are uniformly bounded. Hence $\sup _{h \in \mathbb{N}}\left\|v_{h}\right\|_{\mathrm{BV}(\Omega)}<\infty$ and there exists a subsequence, still denoted by $\left(v_{h}\right)_{h \in \mathbb{N}}$, converging in $\mathrm{L}^{1}(\Omega)$ to a function $u \in \mathrm{BV}(\Omega)$. Obviously, $u=u_{0}$ on $\Omega \backslash \bar{A}$. From the lower semicontinuity of $\overline{F_{p}}$ we finally obtain

$$
\overline{F_{p}}(u, \tilde{A}) \leqslant \liminf _{h \rightarrow \infty} \overline{F_{p}}\left(v_{h}, \tilde{A}\right)=\inf \left\{\overline{F_{p}}(v, \tilde{A}): v=u_{0} \text { on } \Omega \backslash \bar{A}\right\},
$$

and the theorem follows.

Now we can show that the relaxed functional coincides with $F_{p}$ on $\mathrm{C}^{2}$ functions. The proof is based on the geometric lower semicontinuity results of the previous section and on the identity

$$
F_{p}(u, B)=\int_{\mathbb{R}} \int_{\partial\{u \geqslant t\}}\left(1+\left|\mathbf{H}_{\{u \geqslant t\}}\right|^{p}\right) \mathrm{d} \mathcal{H}^{N-1} \mathrm{~d} t \quad \forall u \in \mathrm{C}^{2}(B) .
$$

The identity is a straightforward consequence of the coarea formula and of (7) with $v=\nabla u /|\nabla u|$. 
THEOREM 6 Let $B \subset \Omega$ be an open set and assume that $N \geqslant 2$ and $p>N-1$. The functional $F_{p}(\cdot, B)$ is lower semicontinuous on $\mathrm{L}^{1}(\Omega) \cap \mathrm{C}^{2}(B)$ with respect to the $\mathrm{L}^{1}$ topology. In particular

$$
F_{p}(u, B)=\overline{F_{p}}(u, B) \quad \forall u \in \mathrm{C}^{2}(B) .
$$

Proof. Let $\left(u_{h}\right)_{h \in \mathbb{N}} \subset \mathrm{L}^{1}(\Omega) \cap \mathrm{C}^{2}(B)$ be converging in $\mathrm{L}^{1}(B)$ to $u \in \mathrm{C}^{2}(B)$ and set $L:=$ $\liminf _{h \rightarrow \infty} F_{p}\left(u_{h}, \tilde{A}\right)$, assuming with no loss of generality that $L<\infty$, that the lim inf is a limit and that $u_{h}$ converges a.e. to $u$. By the dominated convergence theorem, $\chi_{\left\{u_{h} \geqslant t\right\}} \rightarrow \chi_{\{u \geqslant t\}}$ in $L^{1}(B)$ whenever $\{u=t\}$ is Lebesgue negligible, hence for a.e. $t \in \mathbb{R}$. In addition, by the Morse Theorem, for almost every $t \in \mathbb{R},\left\{u_{h} \geqslant t\right\}, h \in \mathbb{N}$, and $\{u \geqslant t\}$ have smooth boundaries. Therefore, by applying either Theorem 3 or Theorem 4 we obtain

$$
\int_{\partial\{u \geqslant t\} \cap B}\left(1+\left|\mathbf{H}_{\{u \geqslant t\}}\right|^{p}\right) \mathrm{d} \mathcal{H}^{N-1} \leqslant \liminf _{h \rightarrow \infty} \int_{\partial\left\{u_{h} \geqslant t\right\} \cap B}\left(1+\left|\mathbf{H}_{\left\{u_{h} \geqslant t\right\}}\right|^{p}\right) \mathrm{d} \mathcal{H}^{N-1}
$$

for a.e. $t \in \mathbb{R}$. Integrating over $\mathbb{R}$ and using Fatou's Lemma and 13 yields

$$
F_{p}(u, B) \leqslant \liminf _{h \rightarrow \infty} F_{p}\left(u_{h}, B\right) .
$$

In the two-dimensional case we can say something more about the structure of the solutions. The following theorem is easily deduced from the proofs of Theorems 4.1 and 7.1 in [7]. In particular, it suffices to replace $\partial E$ by $\partial^{*} E$ in the last part of the proof of Theorem 7.1, page 292.

THEOREM 7 ([7]) Let $\Omega \subset \mathbb{R}^{2}$ be an open set and $p>1$. Let $E$ be a Borel set such that there exists a sequence $\left\{E_{h}\right\}_{h \in \mathbb{N}}$ of bounded open sets of class $\mathrm{C}^{2}(\Omega)$ converging to $E$ in $\mathrm{L}^{1}(\Omega)$ and satisfying

$$
\sup _{h \in \mathbb{N}} \int_{\partial E_{h} \cap \Omega}\left(1+|\kappa|^{p}\right) \mathrm{d} \mathcal{H}^{1}<\infty .
$$

Then $E$ has finite perimeter in $\Omega$ and there exists a locally finite family $\Gamma=\left\{\gamma_{i}\right\}_{i \in I}$ of regular curves of class $\mathrm{W}^{2, p}$ such that

1. $\partial^{*} E \cap \Omega \subset \bigcup_{i \in I}\left(\gamma_{i}\right)$;

2. $\Gamma$ is without crossings, i.e. $\frac{d \gamma_{i}\left(t_{1}\right)}{d t} / / \frac{d \gamma_{j}\left(t_{2}\right)}{d t}$ whenever $\gamma_{i}\left(t_{1}\right)=\gamma_{j}\left(t_{2}\right) \in \Omega$ and $t_{1}, t_{2} \in[0,1]$.

Corollary 1 Let $B \subset \Omega \subset \mathbb{R}^{2}$ be an open set and $u \in \mathrm{L}^{1}(\Omega)$ such that $\overline{F_{p}}(u, B)<\infty$, with $p>1$. Then, for almost every $t \in \mathbb{R}$, there exists a locally finite family $\Gamma^{t}=\left\{\gamma_{i}^{t}\right\}_{i \in I_{t}}$ of regular curves of class $\mathrm{W}^{2, p}$ such that $\partial^{*}\{u>t\} \cap B \subset \bigcup_{i \in I_{t}} \gamma_{i}^{t}$ and $\Gamma^{t}$ is without crossings.

Proof. Let $\left\{u_{h}\right\}_{h \in \mathbb{N}} \subset \mathrm{C}^{2}(B)$ be a sequence that converges to $u$ in $\mathrm{L}^{1}(\Omega)$ and a.e. and satisfies $L:=\lim _{h \rightarrow \infty} F_{p}\left(u_{h}, B\right)=\overline{F_{p}}(u, B)<\infty$. Using Fatou's Lemma and 13 we get

$$
\begin{aligned}
\int_{\mathbb{R}} \liminf _{h \rightarrow \infty} \int_{\partial\left\{u_{h}>t\right\} \cap B}\left(1+\left|\mathbf{H}_{\left\{u_{h}>t\right\}}\right|^{p}\right) \mathrm{d} \mathcal{H}^{N-1} & \\
& \leqslant \liminf _{h \rightarrow \infty} \int_{\mathbb{R}} \int_{\partial\left\{u_{h}>t\right\} \cap B}\left(1+\left|\mathbf{H}_{\left\{u_{h}>t\right\}}\right|^{p}\right) \mathrm{d} \mathcal{H}^{N-1}=L<\infty,
\end{aligned}
$$

thus $\liminf _{h \rightarrow \infty} \int_{\partial\left\{u_{h}>t\right\} \cap B}\left(1+\left|\mathbf{H}_{\left\{u_{h}>t\right\}}\right|^{p}\right) \mathrm{d} \mathcal{H}^{N-1}$ is finite for almost every $t \in \mathbb{R}$. The conclusion follows by the application for almost every $t \in \mathbb{R}$ of Theorem 7 possibly passing to a subsequence (depending on $t$ ). 
An obvious consequence of this result is that the same regularity holds for the boundaries $\partial^{*}\{u>t\} \cap \tilde{A}$ (for almost every $t \in \mathbb{R}$ ) of any solution of the disocclusion problem in $\mathbb{R}^{2}$. In addition, since this regularity holds in $\tilde{A}$ and not only within the occlusion $A$, it gives a necessary condition for the existence of a solution, namely that the level lines of the initial function $u_{0}$ must satisfy this regularity property, at least near the boundary of $A$. As a consequence, the only wayessentially - for these level lines to intersect on $\partial A$ is to form a cusp point.

REMARK This regularity result cannot be extended to higher dimensions, due to the fact that controlling the mean curvature does not necessarily guarantee the regularity of a hypersurface. By Allard's regularity theorem (see [1] or Theorem 23.1 in [38]), an $(N-1)$-varifold with density 1 and generalized mean curvature in $\mathrm{L}^{p}, p>N-1$, is supported on a set that can be represented locally as the graph of a $\mathrm{C}^{1,1-(N-1) / p}$ function (J. Duggan [13] showed that $\mathrm{W}^{2, p}$ regularity actually holds). Unfortunately, this regularity does not hold any more in the multiple density case. An example is given in [8] of a varifold $V$ with bounded mean curvature whose support contains a set $A$ of strictly positive measure such that if $a \in A$ then spt $V$ does not correspond to the graph of even a multiple-valued function in any neighborhood of $a$. Thus, controlling only the mean curvature is not enough.

On the other hand, it has been shown by J. Hutchinson [22] that if the second fundamental form of a varifold $V$ is in $\mathrm{L}^{p}, p>N-1$, then $V$ is locally supported on the graph of a multiple-valued $\mathrm{C}^{1,1-(N-1) / p}$ function.

In our disocclusion problem, we can neither ensure that the varifolds supported on the sets $\partial\left\{u_{h}>t\right\}$ converge to unit-density varifolds, nor that the second fundamental form is uniformly bounded in $\mathrm{L}^{p}$, except in the particular case $N=2$ where the mean curvature coincides with the second fundamental form. This explains why our regularity result is stated only for $N=2$.

\section{Acknowledgements}

The work of the first author is part of the European Research Training Network "Homogenization and Multiple Scales" under contract HPRN-2000-00109.

The second author would like to thank Reiner Schätzle and Carlo Mantegazza for the fruitful discussions and their useful comments on this work.

\section{REFERENCES}

1. Allard, W. K. On the first variation of a varifold. Ann. of Math. (2) 95 (1972), 417-491. MR 53 \#1379 MR 46 \#6136 ZZbl 0252.49028

2. Alvarez, L., Gousseau, Y., \& Morel, J.-M. The size of objects in natural and artificial images. Adv. Imaging Electr. Phys. 111 (1999), 167-242.

3. Ambrosio, L., Caselles, V., Masnou, S., \& Morel, J.-M. Connected components of sets of finite perimeter and applications to image processing. J. Europ. Math. Soc. 3 (2001), 39-92. MR 2002g:49072 Zbl 0981.49024

4. Ambrosio, L., Fusco, N., \& Pallara, D. Functions of Bounded Variation and Free Discontinuity Problems. Oxford University Press (2000). MR 2003a:49002 Zbl 0957.49001

5. Ashikhmin, M. Synthesizing natural textures. Proc. ACM Symp. Interactive 3D Graphics (Research Triangle Park, 2001), 217-226. 
6. Ballester, C., Bertalmio, M., Caselles, V., Sapiro, G., \& Verdera, J. Filling-in by joint interpolation of vector fields and gray levels. IEEE Trans. Image Process. 10 (2001), 1200-1211. MR 2003a:94007

7. Bellettini, G., Dal Maso, G., \& Paolini, M. Semicontinuity and relaxation properties of a curvature depending functional in 2D. Ann. Scuola Norm. Sup. Pisa Cl. Sci. (4) 20 (1993), 247-297. MR 94g:49101 Zbl 0797.49013

8. BRAKKe, K. The Motion of a Surface by its Mean Curvature. Princeton University Press (1978). MR 82c:49035

9. Caselles, V., Coll, T., \& Morel, J.-M. Geometry and color in natural images. J. Math. Imaging Vision 2 (2002), 89-105. MR 1892677 Zbl 0994.68167

10. Chan, T. \& Shen, J. Mathematical models for local deterministic inpaintings. SIAM J. Appl. Math. 62 (2001), 1019-1043. Zbl 01730720

11. Cohen, A., Dahmen, W., Daubechies, I., \& DeVore, R. Harmonic analysis of the space BV. Rev. Math. Iberoamericana, submitted (2002).

12. Dal Maso, G. An Introduction to $\Gamma$-Convergence. Progr. Nonlinear Differential Equations Appl. 8, Birkhäuser (1993). MR 94a:49001 Zbl 0816.49001

13. Duggan, J. P. $\mathrm{W}^{2, p}$ regularity for varifolds with mean curvature. Comm. Partial Differential Equations 11 (1986), 903-926. MR 87m:49086|Zbl 0634.35022

14. Efros, A. \& Freeman, W. Image quilting for texture synthesis and transfer. Proc. of SIGGRAPH'01 (Los Angeles, 2001), 341-346.

15. Efros, A. \& Leung, T. Texture synthesis by non-parametric sampling. Internat. Conf. on Comput. Vision, Vol. 2, 1999, 1033-1038.

16. EULER, L. Methodus inveniendi lineas curvas maximi minimive proprietate gaudentes. Lausanne (1744). Zbl 0788.01072

17. Evans, L. C. \& Spruck, J. Motion of level sets by mean curvature II. Trans. Amer. Math. Soc. 330 (1991), 635-681. MR 92f:58050 Zbl 0829.53040

18. Fantoni, C. \& Gerbino, W. Probing the amodal completion of asymmetric occluded angles: a test of interpolation models. J. Vision 1 (2001), 461a; available at http://journalofvision.org/1/3/461.

19. Federer, H. Geometric Measure Theory. Springer (1969). MR 41 \#1976 Zbl 0874.49001

20. Giusti, E. Minimal Surfaces and Functions of Bounded Variation. Birkhäuser (1994). MR 87a:58041 Zbl 0545.49018

21. Horn, B. K. P. The curve of least energy. ACM Trans. Math. Software 9 (1983), 441-460. MR 87c:65009|Zbl 0533.41007

22. HUtChinson, J. E. $\mathrm{C}^{1, \alpha}$ multiple function regularity and tangent cone behaviour for varifolds with second fundamental form in $\mathrm{L}^{p}$. Geometric Measure Theory and the Calculus of Variations (Arcata, CA, 1984), W. K. Allard and F. J. Almgren (eds.), Proc. Sympos. Pure Math. 44, Amer. Math. Soc. (1986), 281-306. MR 87i:49059ZZbl 0635.49020

23. Kanizs A, G. Organisation in Vision. Praeger, New York (1979).

24. Leung, T. \& MALiK, J. Contour continuity in region based image segmentation. Fifth Euro. Conf. Computer Vision (Freiburg, 1998), 1, 544-559.

25. Liang, L., LiU, C., Xu, Y., Guo, B., \& Shum, H.-Y. Real-time texture synthesis by patch-based sampling. Microsoft Research Technical Report, MSR-TR-2001-40, March 2001.

26. Masnou, S. Filtrage et désocclusion d'images par méthodes d'ensembles de niveau. PhD thesis, Université Paris-Dauphine (1998).

27. Masnou, S. Disocclusion: a variational approach using level lines. IEEE Trans. Image Process. 11 (2002), 68-76. MR 2002m:68111

28. Masnou, S. \& Morel, J.-M. Level lines based disocclusion. Proc. ICIP'98 IEEE Internat. Conf. on Image Processing (Chicago, 1998), Vol. 3, 259-263. 
29. Masnou, S. \& Morel, J.-M. Construction of solutions to the disocclusion problem. Forthcoming (2002).

30. Mumford, D. Elastica and computer vision. Algebraic Geometry and Applications, C. Bajaj (ed.), Springer (1994), 491-506. MR 95a:92026 Zbl 0798.53003

31. Nitzberg, M., Mumford, D., \& Shiota, T. Filtering, Segmentation and Depth. Lecture Notes in Comput. Sci. 662, Springer (1993). MR 94h:92012 Zbl 0801.68171

32. Paget, R. \& Longstaff, I. Texture synthesis via a noncausal nonparametric multiscale Markov random field. IEEE Trans. Image Process. 7 (1998), 925-931.

33. Popat, A. C. Conjoint probabilistic subband modeling. PhD thesis, Massachussets Institute of Technology (1997).

34. Rudin, L. \& Osher, S. Total variation based image restoration with free local constraints. Proc. IEEE ICIP'94 (Austin, TX, 1994), Vol. 1, 31-35.

35. SCHÄTZLE, R. Hypersurfaces with mean curvature given by a trace. Habilitationsschrift, AlbertLudwigs-Universität, Freiburg im Breisgau (1999).

36. SchätZle, R. Quadratic tilt-excess decay and strong maximum principle for varifolds. Preprint (2000).

37. Sharon, E., Brandt, A., \& Basri, R. Completion energies and scale. Proc. IEEE Conf. Comput. Vision and Pattern Recognition (San Juan, Puerto Rico, 1997), 884-890.

38. Simon, L. Lectures on Geometric Measure Theory. Proc. Centre for Math. Anal. 3, Australian Nat. Univ. (1983). MR 87a:49001 Zbl 0546.49019

39. Thornber, K. K. \& Williams, L. R. Analytic solution of stochastic completion fields. Biological Cybernetics 75 (1996), 141-151. Zbl 0869.92030

40. Thornber, K. K. \& Williams, L. R. Characterizing the distribution of completion shapes with corners using a mixture of random processes. Pattern Recognition 33 (2000), 543-553.

41. Ullman, S. Filling-in the gaps: the shape of subjective contour and a model for their generation. Biological Cybernetics 25 (1976), 1-6.

42. WeI, L.-Y. \& Levoy, M. Fast texture synthesis using tree-structured vector quantization. Proc. SIGGRAPH 2000, 479-488.

43. Williams, L. R. \& JACOBS, D. W. Stochastic completion fields: a neural model of illusory contour shape and salience. Neural Computation 9 (1997), 837-858.

44. ZHU, S., WU, Y., \& MUMFORD, D. Filters, random fields and maximum entropy (FRAME)—towards a unified theory for texture modeling. Internat. J. Comput. Vision 27 (1998), 107-126.

45. ZIEMER, W. P. Weakly Differentiable Functions. Springer (1989). MR 91e:46046Zbl 0692.46022 